\title{
Plastic plastids
}

\section{Peter Nick}

(C) Springer-Verlag Wien 2014

Science is reputed to be objective, and it appears that do not leave much space for passionate debates. However, sometimes, such debates arise, especially, when observation approaches the limits of that what is observable. We should be grateful for such cases, because insight and understanding require discussion and even controversy to develop. The recent debate on the mutual relationship between plastids provides a neat example to illustrate this point. Whereas plastids were traditionally described as semi-autonomous entities dwelling independently in their hosting plant cell, advances in microscopy have led to the concept of interconnected plastids. This new concept is mainly based (and in turn has stimulated) a reinterpretation of classical reports on protrusions from the plastid surface, so called stromules, as bridges linking different plastids. Surely, the model that these important organelles are organised as a cross-communicating population of domesticated, organism-like entities, is tantalising. No wonder, that this new viewpoint acquired a degree of popularity which made it enter into textbooks soon after. However, although inspiring, the actual evidence for plastid interconnectedness has remained scarce and the interpretation of stromules as interconnecting bridges between individual plastids, despite being plausible at first sight, might turn out to be a case, where an admittedly charming interpretation has overwhelmed that what is actually seen under the microscope.

In their comprehensive review in the current issue, Schattat et al. (2015) critically discuss the concept of the interconnected plastid and rehabilitate the original model of plastids as unitary, discrete units. However, as should be the goal of all serious discussion, they offer a new explanation for the observations leading to the concept of interconnectivity and embed

P. Nick $(\bowtie)$

Botanical Institute, Karlsruhe Institute of Technology, Karlsruhe, Germany

e-mail: peter.nick@kit.edu this explanation into the historical context of research on plastids. This is science at its best: explain and integrate to reach a deeper level of insight. The debate on stromules and interconnectivity moves at the borderline of that what we can observe, and therefore, this debate has always been strongly shaped by advances in methodology. This point is far from trivial - it reminds us of the fact that our concepts depend on our methods. After briefly introducing the historical context leading to the concept of the discrete plastid, the authors give a survey on the five decades of research on stromules that also left footprints in this journal - the most detailed and highquality description of stromule dynamics and behaviour is probably that given in Gunning (2005). The fact that stromules exist, has meanwhile been shown beyond doubt for numerous types of plastids and organisms. That they can reach from one plastid to the other has also been observed many times. However, the crucial question remains, whether they really represent bridges, where two previously distinct compartments are fused to a new entity. When we look into the microscope, we basically perceive projections of the threedimensional reality into a two-dimensional image. Advances of fluorescence microscopy have allowed to improve our resolution into the third dimension and thus to improve our spatial representation of cells. However, stromules are located at the borderline of resolution, which for confocal microscopy is in the range of half a micron.

The decisive criterion for interconnectivity is, whether the observed bridges between plastids can transport molecules that otherwise should not be able to cross a membrane. This criterion has been addressed, in a crucial experiment, in leucoplasts of tobacco roots, where the green fluorescent protein was targeted to the stroma (Köhler et al. 1997). When in apparently interconnected pairs of plastids, this reporter was photobleached in one half, the bleached region recovered, obviously by import of the reporter from the other, unbleached half. This observation documenting structural contiguity of 
the irradiated pair was seminal for the concept of plastid interconnectivity. As a drawback of the photobleaching approach, the region of interest is transiently invisible as consequence of bleaching (however, the observability is restored subsequently by the migration of the fluorescent reporter into the previously bleached region). The team of Jaideep Mathur had developed alternative strategies based on novel photoconvertible reporters, where bleaching is replaced by a colour change. This allows to observe dynamic changes continuously. When they applied this technique to stromules, they found, unexpectedly, that the converted reporter did not cross from one plastid to the other (Schattat et al. 2012), which is discordant with the model of stromules as manifestations of plastid interconnectivity.

A situation, where two observations are completely discrepant, might indicate that experimental artefacts are responsible for the controversy. However, as stringently elaborated by the authors, the stromule case is much more interesting, because it is a matter of interpretation and terminology rather than a matter of experimental drawbacks: The central point is that the term (and the concept) of a stromule has been used in an ambiguous manner. Whereas originally designating protrusions from the surface of plastids, it has later been extended to the filamentous, bridge-like structures of apparently interconnected plastids. However, as pointed out by the authors, such bridge-like structures can be formed during the later phases of plastid division, especially in the non-chloroplastic forms of plastids, such as etioplasts or leucoplasts. Although this fact had been known for a long time - the seminal paper by Reinhard (1933) was published in one of the first volumes of this journal-it had shifted a bit out of focus later.

Thus, correct terminology is important to come to correct concepts. The authors demonstrate convincingly that the idea of interconnected plastids was based on a misinterpretation of interplastidic bridges probably representing late stages of plastid division. Although it may seem disappointing to discard this fascinating idea, the debate has brought us a fascinating new question: when plastids are so plastic, and when this plasticity is not manifestation of their continuity, what then is the function of stromules and what are the mechanisms that make them appear?

Conflict of interest The author declares that there is no conflict of interest.

\section{References}

Gunning BES (2005) Plastid stromules: video microscopy of their outgrowth, retraction, tensioning, anchoring, branching, bridging, and tip-shedding. Protoplasma 225:33-42

Köhler RH, Cao J, Zipfel WR, Webb WW, Hanson MR (1997) Exchange of protein molecules through connections between higher plant plastids. Science 276:2039-2042

Reinhard H (1933) Über die Teilung der Chloroplasten. Protoplasma 19: 541-564

Schattat MH, Griffiths S, Mathur N, Barton K, Wozny MR, Dunn N et al (2012) Differential coloring reveals that plastids do not form networks for exchanging macromolecules. Plant Cell 24:1465-1477

Schattat M, Barton K, Mathur J (2015) The myth of interconnected plastids and related phenomena. Protoplasma, current issue 